\title{
COMPARISON OF DIFFERENT ATTITUDE CORRECTION MODELS FOR ZY-3 SATELLITE IMAGERY
}

\author{
W. P. Song ${ }^{1}$, S. J. Liu ${ }^{1, *}$, X. H. Tong ${ }^{1}$, C. L. Niu ${ }^{2}$, Z. Ye ${ }^{1}$, H. Zhang ${ }^{1}$, Y. M. Jin ${ }^{1}$ \\ ${ }^{1}$ College of Surveying and Geo-Informatics, Tongji University, 1239 Siping Road, Shanghai, China - \\ liusjtj@tongji.edu.cn \\ ${ }^{2}$ Qingdao Surveying \& Mapping Institute, Qingdao, China-chlniu@163.com
}

Commission III, ICWG III/IVb

KEY WORDS: Systematic Errors, Attitude Quaternion, Rigorous Model, Attitude Angle, ZY-3 Satellite

\begin{abstract}
:
ZY-3 satellite, launched in 2012, is the first civilian high resolution stereo mapping satellite of China. This paper analyzed the positioning errors of ZY-3 satellite imagery and conducted compensation for geo-position accuracy improvement using different correction models, including attitude quaternion correction, attitude angle offset correction, and attitude angle linear correction. The experimental results revealed that there exist systematic errors with ZY-3 attitude observations and the positioning accuracy can be improved after attitude correction with aid of ground controls. There is no significant difference between the results of attitude quaternion correction method and the attitude angle correction method. However, the attitude angle offset correction model produced steady improvement than the linear correction model when limited ground control points are available for single scene.
\end{abstract}

\section{INTRODUCTION}

High-precise positioning is an important guarantee for mapping multi-scale topographic maps. However, a little satellite variation and device loss\&aging will change sensor parameters which will bring systematic errors. Therefore, the way to eliminate systematic errors effectively, including satellite attitude-angle errors and sensor imaging parameters errors, have a great significance to improve the geo-position accuracy.

As for high-resolution satellite, the positioning accuracy without control points or with scarce control points has reached a very high level over the years. As the first aerospace three-array sensor, the positioning model of Germany's MOMS-02 has laid a foundation for the following study (Kornus et al., 2006). Toutin did some experiments which show that the positioning accuracy kept stabilized with the reduction of control points (Toutin et al., 2006). And now, the series imagery such as SPOT, IKONOS and QUICKBIRD have formed relatively complete positioning systems (Eisenbeiss $\mathrm{H}$ et al., 2006). At present, WordView-3 satellite has already achieved $0.31 \mathrm{~m}$ geoposition accuracy (Longbotham et al. 2014). In China, the correctness of rigorous equations, established using six Kepler parameters and three sensor parameters, is verified using SPOT imagery (Yuan et al., 2012). Based on Mapping Satellite-1, Wang et al. have done in-depth researches and analysis mainly on camera geometric calibration, equivalent frame photo (EFP) beam adjustment, low-frequency error compensation and other key issues (Wang et al., 2013; Wang et al., 2014; Wang et al., 2016). Li et al. eliminated systematic errors effectively using spatial aerotriangulation of EFP beam adjustment method (Li et al.,2012). The systematic errors in the elements of exterior orientation was eliminated more effectively by combining the elements of exterior orientation and self-calibration parameters (Zhang et al., 2015). However, the direct positioning accuracy of most domestic satellites are difficult to meet demand of highprecision positioning, and still many problems shall be solved urgently because of the limitations of hardware level, orbit determination and so on (Gu et al., 2012).
ZY-3 satellite, launched in 2012, entered scheduled track successfully and got the first batch of three-dimensional mapping imagery, which have filled gaps in the field of stereoscopic mapping (Li et al., 2012). As the first civilian high resolution stereo mapping satellite of China, ZY-3 satellite was with two forward-looking and backward-looking full color TDI CCD cameras whose resolutions are all better than $3.5 \mathrm{~m}$, one nadir-looking full color TDI CCD camera whose resolution is better than $2.1 \mathrm{~m}$, and one multi-spectral camera whose resolution is better than $5.8 \mathrm{~m}$. The rigorous center-projection relationship exists between each scanning line imagery and ground points, which can be analyzed from ZY-3 satellite auxiliary data such as flight attitude data, track data and so on. $\mathrm{Li}$ et al. built the rigorous model based on ZY-3 satellite imagery, and achieved better than $15 \mathrm{~m}$ non-control positioning accuracy in plane, and $3 \mathrm{~m}$ in height and $4 \mathrm{~m}$ in plane positioning accuracy with control (Li et al., 2012).

Motivation of this study is to further eliminate systematic errors contained in satellite attitude-angle and sensor imaging parameters effectively. The rest of this paper is structured as follows. In Section II, we describe study datasets and give some backgrounds of ZY-3 satellite. In Section III, the basic models, such as rigorous model, attitude quaternion correction and attitude-angle correction, are introduced. In Section IV, the experimental methods based on ZY-3 satellite imagery and the built models are presented. And the results derived from different experimental methods are evaluated and an in-depth discussion is carried out. In Section V, we draw our conclusions.

\section{DATASET}

In this paper, all experiments are based on the same-track threearray forward-looking and backward-looking imagery of ZY-3 satellite in Songshan area (Liu et al., 2016). The image capture time was February 3, 2012, the raw forward-looking and backward-looking imagery are composed of 4 chips CCDs, and each piece size of CCD imagery is $4096 \times 16384$. A total of 42 control points are actually measured by GPS, with an accuracy of better than $5 \mathrm{~cm}$, and mostly distributed in road crosses or corners where are easy to identify. In the experiments, we use 
the measured points as true values to detect ZY-3 satellite positioning accuracy. The distribution of the points measured by GPS is shown in Figure 1.

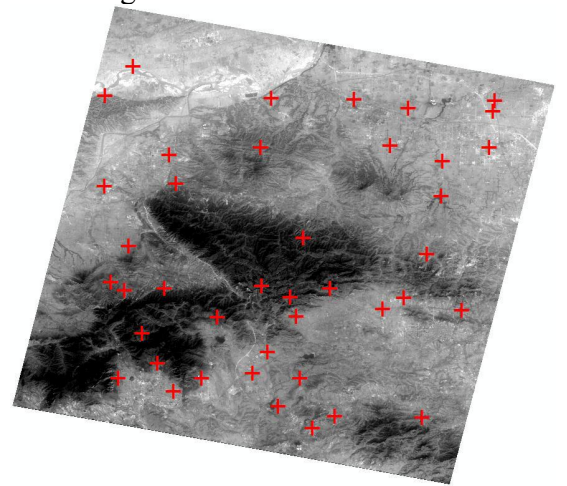

Figure 1. The distribution of the points measured by GPS (Liu et al., 2016)

\section{MODELS}

ZY-3 satellite sensor is a satellite-borne three-array CCD sensor whose focal length is $f$. According to the collinearity equation, ground points are converted to image space so as to establish the relationship between image points and ground points. If the spatial shift of GPS antenna phase and sensor lens center in satellite body system are considered, and without considering the factors such as atmospheric refraction, attitude matrix placement errors, rigorous model of ZY-3 satellite can be shown in the literature(Zhang et al. 2016), which describe the relationship between ground point coordinates in WGS84 coordinate system and image points using scale factors, the sensor coordinates, spatial shift of GPS antenna phase, spatial shift of sensor lens center in satellite body coordinate system and different rotation matrices (Zhang et al. 2016). In this paper, the quadratic polynomial model is used to fit orbital elements of exterior orientation, including position and attitude. So the external orientation elements of every scanning line are the functions of scanning time which can reduce the number of unknowns, and the solution of the normal equation will be more stable.

As for ZY-3 satellite, two models can be used to describe its attitude, one is attitude quaternion correction model, the other is attitude angle correction model. As for attitude quaternion correction model, because attitude quaternion for ZY-3 satellite are used to describe the motion attitudes, it is of great significance to use attitude quaternion to correct attitude systematic errors. Firstly, rigorous model can be describe by looking angles which is nonlinear. And then the rigorous model described by looking angles shall be normalized. Finally, after the linearization, the results of normalization equation can be shown in the literature (Jia et al., 2014).

Attitude angle correction model is to correct three sensor attitude angles (Roll, Pitch, Yaw). And systematic errors can be either constant or linear. Due to the high orbit-determination accuracy of ZY-3 satellite, orbit data obtained from GPS can be used as true values. So in this paper, the systematic errors of attitude data shall be the only considered. Generately, attitudeangle correction model can be divided into two parts (Zhang et al, 2014). (a) Systematic error of attitude angle is viewed as a constant. In this case, systematic errors contained in attitude angles are described using constant. (b) Systematic errors of attitude angle is viewed as the linear function of time $t$, which is called linear correction model. In linear correction model, each control point can set two error equations. When the number of control points is no less than 3, the systematic error parameters of attitude angle can be solved according to the least squares principle. According to these parameters and the scanning line time, higher-precision attitude angle without systematic errors can be solved.

\section{EXPERIMENTS AND DISCUSSIONS}

\subsection{Stereo positioning after element corrections of exterior orientation}

As for array satellite imagery, orientation elements of every scanning line can not be calculated theoretically. So suitable mathematical models shall be applied to fit external orientation elements. The main mathematical models include quadratic polynomial model, systematic error compensation model and oriented slice model. In this experiment, quadratic polynomial model is selected. So the stereo positioning experiment is based on rigorous model using ZY-3 satellite imagery as auxiliary data for analysis, and 42 measured control points from GPS. In order to eliminate attitude measurement errors, equipment installation errors et al., the calibration parameters of offset matrix is solved from ground check-field (Tang et al., 2012). Exterior orientation elements are re-modeled by quadratic polynomial model. The main process is performed as follows: (a) Re-project ground points from ground space to image space, and then check the accuracy in image space. (b) Stereoscopic positioning using forward-looking and backward-looking imagery, and then check ZY-3 satellite stereo positioning accuracy without control points using quadratic polynomial model.

Table 1 lists maximum residuals, minimum residuals and RMSE. Table 2 lists non-control stereoscopic positioning results, including maximum residuals and RMSE. In Table 1 and Table 2, quadratic polynomial model is abbreviated as QPM, maximum error is abbreviated as $\mathrm{E}_{\mathrm{Max}}$.

\begin{tabular}{|c|c|c|c|c|c|}
\hline Model & Statistic & \multicolumn{2}{|c|}{$\begin{array}{l}\text { Forward } \\
\text { looking }\end{array}$} & \multicolumn{2}{|c|}{$\begin{array}{l}\text { Backward } \\
\text { looking }\end{array}$} \\
\hline QPM & $\begin{array}{l}\text { E }_{\text {Max }} / \text { pixel } \\
\text { RMSE/pixel }\end{array}$ & $\begin{array}{l}\text { Row } \\
5.42 \\
3.37\end{array}$ & $\begin{array}{l}\text { Column } \\
3.66 \\
2.34\end{array}$ & $\begin{array}{l}\text { Row } \\
5.55 \\
3.67\end{array}$ & $\begin{array}{l}\text { Column } \\
3.94 \\
2.57\end{array}$ \\
\hline
\end{tabular}

Table 1. Re-projection residuals of QPM

\begin{tabular}{|c|c|c|c|c|}
\hline Model & Statistic & \multicolumn{3}{|c|}{ WGS 84 coordinate system } \\
\hline \multirow{3}{*}{ QPM } & & $\mathrm{X}$ & $\mathrm{Y}$ & $\mathrm{Z}$ \\
\hline & $\mathrm{E}_{\mathrm{Max}} / \mathrm{m}$ & 13.61 & 16.84 & 21.68 \\
\hline & $\mathrm{RMSE} / \mathrm{m}$ & 7.39 & 8.32 & 7.57 \\
\hline
\end{tabular}

Table 2. Stereo positioning accuracy of QPM

From Table 2, we can see the stereo positioning accuracy are not very good and exist significant systematic errors which should be eliminated before we use them. The results are as follows: a. As for forward-looking imagery, RMSE of reprojection are 3.37 pixels in flight direction, 2.34 pixels in scanning direction. b. As for backward-looking imagery, RMSE of direct re-projection are 3.67 pixels in flight direction, 2.57 pixels in scanning direction. RMSE of stereoscopic positioning are $7.39 \mathrm{~m}$ in $\mathrm{X}$ direction, $8.32 \mathrm{~m}$ in $\mathrm{Y}$ direction and $7.57 \mathrm{~m}$ in height.

\subsection{Stereo positioning after attitude quaternion correction}

In order to ensure calculation stability, no less than 3 control points are selected. And the higher the accuracy of measured 
control points are, the more accurate the modified values of attitude quaternion are, and the higher the positioning accuracy after attitude quaternion systematic error correction is. The main steps can be seen as follows: (a) Use spherical interpolation method to interpolate the quaternion corresponding to the measured control points; (b) By using 4, 10, 20 and 42 control points respectively, we calculate the attitude quaternion, while analyze the effect caused by the number of control points, and then carry out the stereoscopic positioning.

Table 3 are the positioning results after the correction of attitude, which is always using the 42 GPS points as check points.

\begin{tabular}{|c|l|l|c|l|}
\hline $\begin{array}{c}\text { Control } \\
\text { points }\end{array}$ & \multicolumn{4}{|c|}{$\mathrm{RMSE} / \mathrm{m}$} \\
\hline & $\mathrm{RMS}_{\mathrm{X}} / \mathrm{m}$ & $\mathrm{RMS}_{\mathrm{Y}} / \mathrm{m}$ & $\mathrm{RMS}_{\mathrm{XY}} / \mathrm{m}$ & $\mathrm{RMS}_{\mathrm{Z}} / \mathrm{m}$ \\
4 & 4.69 & 4.35 & 6.40 & 3.27 \\
10 & 4.93 & 4.12 & 6.42 & 3.09 \\
20 & 4.71 & 3.42 & 5.82 & 4.79 \\
42 & 4.59 & 3.93 & 6.04 & 3.11 \\
\hline
\end{tabular}

Table 3. The stereo positioning accuracy after attitude quaternion correction

In this paper, attitude quaternion corrections are described using $\mathrm{dq}_{0}, \mathrm{dq}_{1}, \mathrm{dq}_{2}$, and $\mathrm{dq}_{3}$. From experiment 4.2 , we can see no matter the forward-looking imagery or the backward-looking imagery, the corrections $\mathrm{dq}_{1}, \mathrm{dq}_{2}$ of the attitude quaternion systematic errors are larger one order of magnitude than $\mathrm{dq}_{0}, \mathrm{dq}_{3}$ And also with the increase of control points, systematic error corrections of attitude quaternion have no really large differences and gradually stabilize. Compare the results of experiment 4.1 , we can conclude the positioning accuracy can be improved after attitude correction with aid of ground controls. And different numbers of control points have no significant effect on the corrections of attitude quaternion systematic errors. After corrections, the positioning accuracy is about $6 \mathrm{~m}$ in plane, and $3 \mathrm{~m}$ in height.

\subsection{Stereo positioning after attitude angle correction}

Attitude angle correction is to correct three sensor attitude angles (Roll, Pitch, Yaw). The systematic errors can be either constant or linear. So constant angular model and linear model of attitude angles are selected, and then compare these two models using 42 GPS points. The stereo positioning results after constant angular model correction are in Table 4, Table 5 lists stereo positioning residuals without control points after linear model correction.

\begin{tabular}{|c|c|c|c|c|}
\hline $\begin{array}{l}\text { Control } \\
\text { points }\end{array}$ & \multicolumn{4}{|c|}{$\mathrm{RMSE} / \mathrm{m}$} \\
\hline & $\mathrm{RMS}_{\mathrm{X}} / \mathrm{m}$ & $\mathrm{RMS}_{\mathrm{Y}} / \mathrm{m}$ & $\mathrm{RMS}_{\mathrm{XY}} / \mathrm{m}$ & $\mathrm{RMS}_{\mathrm{Z}} / \mathrm{m}$ \\
4 & 2.73 & 4.93 & 5.64 & 4.11 \\
10 & 2.73 & 4.67 & 5.41 & 4.79 \\
20 & 2.73 & 4.69 & 5.43 & 4.03 \\
42 & 2.62 & 4.61 & 5.30 & 4.02 \\
\hline
\end{tabular}

Table 4. Stereo positioning results after constant angular model correction

\begin{tabular}{|c|c|c|c|c|}
\hline $\begin{array}{l}\text { Control } \\
\text { points }\end{array}$ & \multicolumn{4}{|c|}{ RMSE/m } \\
\hline & RMS $_{X} / \mathrm{m}$ & RMSY/m & RMS $_{\mathrm{XY}} / \mathrm{m}$ & $\mathrm{RMS}_{\mathrm{Z}} / \mathrm{m}$ \\
4 & 2.92 & 6.95 & 7.54 & 7.26 \\
10 & 2.71 & 3.42 & 4.36 & 6.01 \\
20 & 2.73 & 3.61 & 4.53 & 3.99 \\
42 & 2.67 & 3.36 & 4.29 & 3.94 \\
\hline
\end{tabular}

Table 5. Stereo positioning results after linear model correction
From Table 4 and Table 5, we can find the positioning accuracy can be improved greatly after the corrections of attitude angle systematic errors, and the correction effects of constant angular model is better than that of linear model of attitude angle for single ZY-3 satellite imagery. The results are as follows: (a). As for the correction of constant angular model, the positioning accuracy is increased from $5.64 \mathrm{~m}$ to $5.30 \mathrm{~m}$ in plane and $4.79 \mathrm{~m}$ to $4.02 \mathrm{~m}$ in height with the increase of control points on the whole. (b) As for the correction of linear model, the positioning accuracy is increased from $7.54 \mathrm{~m}$ to $4.29 \mathrm{~m}$ in plane and from $7.26 \mathrm{~m}$ to $3.94 \mathrm{~m}$ in height with the increase of control points on the whole.

\section{CONCLUSIONS}

Based on the original researches and supported by ZY-3 threeline-array stereoscopic mapping imagery, this paper studies the methods of eliminating systematic errors caused by satellite attitude-angle and sensor imaging parameters effectively through further experiments. The experimental results revealed the following:

(a) The stereo positioning accuracy using quadratic polynomial are not very good and there exist significant systematic errors when using ZY-3 satellite imagery for stereoscopic positioning.

(b) As for these ZY-3 satellite images, with the increase of control points, systematic error corrections of attitude quaternion have no really large differences and gradually stabilize. And the positioning accuracy can be improved after attitude correction with aid of ground controls.

(c) Compared constant angular model and linear model of attitude angle, the positioning accuracy based on constant angular model and linear model of attitude angle are better than the cases of quadratic polynomial model. And there is no significant difference between the results of attitude quaternion correction method and the attitude angle correction method. However, attitude angle offset correction model produced steady improvement than linear correction model when limited ground control points are available. And the use of constant angular model of attitude angle is more in line with the law of attitude changes than linear model of attitude angle for single scene.

\section{ACKNOWLEDGEMENTS}

The authors would like to thank the editor and anonymous reviewers for their valuable comments on the improvement of the paper. This paper was substantially supported by the National Natural Science Foundation of China (Project Nos. 41631178, 41325005, 41401531 and 41601414), and the Fundamental Research Funds for the Central Universities.

\section{REFERENCES}

Eisenbeiss H, Baltsavias E, Pateraki M, et al. 2004. Potential of IKONOS and QUICKBIRD imagery for accurate 3D-Point positioning, orthoimage and DSM generation. International Archives of the Photogrammetry, Remote Sensing and Spatial Information Sciences, 35(B3): 522-528.

Kornus W, Alamús R, Ruiz A, et al. 2006. DEM generation from SPOT-5 3-fold along track stereoscopic imagery using autocalibration. ISPRS Journal of Photogrammetry and Remote Sensing, 60(3): 147-159. 
$\mathrm{Gu}$ X. 2012. Principle and method of radiometric calibration for space optical remote sensing, Beijing: Science Press.

Jia B, Jiang T, Yu A. Z. 2014. A method of systematic error compensation of attitude quaternion for ZY-3 satellite. Engineering of Surveying and Mapping, 23(5):10-12.

Li D. 2012. China's first civilian three-line-array stereo mapping satellite: ZY-3. Acta Geodaetica et Cartographica Sinica, 41(3): 317-322

Wang R, Wang J, Hu X. 2016. Preliminary location accuracy assessments of $3^{\text {rd }}$ satellite of TH-1. Acta Geodaetica Et Cartographica Sinica. 45(10):1135-1139.

Wang R, Wang J, Hu X. 2014. The EFP bundle adjustment of all three line intersection. Editorial Board of Geomatics and Information Science of Wuhan University, 39(7):757-761.

Wang R, Hu X, Wang J. 2013. Photogrammetry of mapping satellite-1 without ground control points. Acta Geodaetica Et Cartographica Sinica, 42(1):1-5.

Longbotham N, Pacifici F, Baugh B, et al. 2014. Prelaunch assessment of worldview-3 information content. Hyperspectral Image and Signal Processing: Evolution in Remote Sensing (WHISPERS), 2014 6th Workshop on. IEEE: 1-4.

Toutin T. 2006. Generation of DSMs from SPOT-5 in-track HRS and across-track HRG stereo data using spatiotriangulation and autocalibration. ISPRS Journal of Photogrammetry and Remote Sensing, 60(3): 170-181.

Yuan X, Yu X. 2012. Calibration of angular systematic errors for high resolution satellite imagery. Acta Geodaetica Et Cartographica Sinica. 42(3):385-392.

Tang X, Zhang G, Zhu X, et al. 2013. Triple linear-array image geometry model of ZiYuan-3 surveying satellite and its validation. International Journal of Image and Data Fusion, 4(1): $33-51$

Liu S, Tong X, Wang F, et al. Attitude Jitter Detection Based on Remotely Sensed Images and Dense Ground Controls: A Case Study for Chinese ZY-3 Satellite. 2016. IEEE Journal of Selected Topics in Applied Earth Observations and Remote Sensing, 9(12): 5760-5766 